\title{
Detecting emotion from EEG signals using the emotive epoc device
}

CONFERENCE PAPER · DECEMBER 2012

DOI: 10.1007/978-3-642-35139-6_17

CITATIONS

2

2 AUTHORS, INCLUDING:

\section{Rafael Ramirez}

University Pompeu Fabra

71 PUBLICATIONS 248 CITATIONS

SEE PROFILE
READS

327 


\title{
Detecting Emotion from EEG Signals Using the Emotive Epoc Device
}

\author{
Rafael Ramirez and Zacharias Vamvakousis \\ Department of Information and Communication Technologies \\ Universitat Pompeu Fabra \\ Roc Boronat 138, 08018 Barcelona, Spain \\ \{rafael.ramirez, zacharias .vamvakousis\}@upf .edu
}

\begin{abstract}
The study of emotions in human-computer interaction has increased in recent years in an attempt to address new user needs. At the same time, it is possible to record brain activity in real-time and discover patterns to relate it to emotional states. This paper describes a machine learning approach to detect emotion from brain activity, recorded as electroencephalograph (EEG) with the Emotic Epoc device, during auditory stimulation. First, we extract features from the EEG signals in order to characterize states of mind in the arousal-valence $2 \mathrm{D}$ emotion model. Using these features we apply machine learning techniques to classify EEG signals into high/low arousal and positive/negative valence emotional states. The obtained classifiers may be used to categorize emotions such as happiness, anger, sadness, and calm based on EEG data.
\end{abstract}

\section{Introduction}

The study of emotions in human-computer interaction has increased in recent years. This is due to the growing need for computer applications capable of detecting the emotional state of users [17. Motivated by every day interaction among humans, a great part of the research in this area has explored detecting emotions from facial and voice information. Under controlled situations, current emotion-detection computer systems based on such information are able to classify emotions with considerable accuracy [18. However, emotions are not always manifested by means of facial expressions and voice information. Psychologists distinguish between physiological arousal, behavioral expression, and the conscious experience of emotions. Facial and voice information is related only to behavioral expression which can be consciously controlled and modified, and which interpretation is often subjective. Thus, other approaches to detect emotion have been proposed which focus on different physiological information such as heart rate, skin conductance, and pupil dilation [18]16. A still relatively new field of research in affective brain-computer interaction attempts to detect emotion using electroencephalograms (EEGs) [2]3. There have been several approaches to EEG-based emotion detection, but there is still little consensus about definite conclusions. 
In this paper we describe an approach to detecting emotion from electroencephalogram signals measured with a (low-cost) Emotiv EPOC headset. We present to subjects auditory stimuli from a library of emotion-annotated sounds and record their response EEG activity. We then filter and process the signal in order to extract emotion-related features and apply machine learning techniques to classify emotional states into high/low arousal and positive/negative valence (e.g. happiness is a state with high arousal and positive valence, whereas sadness is a state with low arousal and negative valence). Our approach differs from previous works in that we do not rely in subject self-reported emotional states during stimuli presentation. Instead, we use a library of emotion-annotated sounds publicly available for emotional research. Figure 1 illustrates the different steps of our approach.

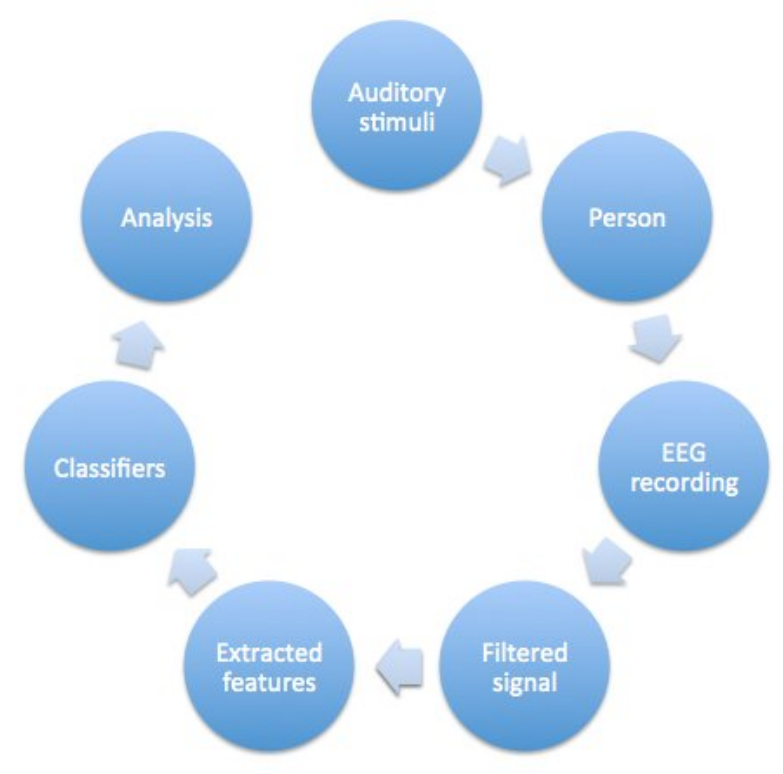

Fig. 1. Schematic view of the system

The rest of the paper is organized as follows: Section 2 presents background to this research. Section 3 described the data collection process and methods. In Section 4 we describe our approach to EEG-based emotion classification and report the results, and finally Section 5 presents some conclusions and further research.

\section{Background}

The firing of neurons in the brain trigger voltage changes. The electrical activity measured by the electrodes in an EEG headset corresponds to the field potentials resulting from the combined activity of many individual neuronal cells in the 
brain cortex. However, the measured cortical activity is distorted by the tissue and skull between the electrodes and the neurons. This introduces noise and reduce the intensity of the recorded signals. In despite of this, EEG measurements still provide important insight into the electrical activity of the cortex.

The frequency of EEG measurements ranges from 1 to $80 \mathrm{~Hz}$, with amplitudes of 10 to 100 microvolts 9 . Signal frequencies have been divided into different bands, since specific frequency waves are normally more prominent in particular states of mind. The two most important frequency waves are the alpha waves $(8-12 \mathrm{~Hz})$ and the beta waves $(12-30 \mathrm{~Hz})$. Alpha waves predominantly originate during wakeful relaxation mental states, and are most visible over the parietal and occipital lobes. Intense alpha wave activity have also been correlated to brain inactivation. Beta wave activity, on the other hand, is related to an active state of mind, most prominent in the frontal cortex during intense focused mental activity [9].

Alpha and beta wave activity may be used in different ways for detecting emotional (arousal and valence) states of mind in humans (more details later). Choppin [3] propose to use EEG signals for classifying six emotions using neural networks. Choppin's approach is based on emotional valence and arousal by characterizing valence, arousal and dominance from EEG signals. He characterize positive emotions by a high frontal coherence in alpha, and high right parietal beta power. Higher arousal (excitation) is characterized by a higher beta power and coherence in the parietal lobe, plus lower alpha activity, while dominance (strength) of an emotion is characterized as an increase in the beta / alpha activity ratio in the frontal lobe, plus an increase in beta activity at the parietal lobe.

Oude 14 describes an approach to recognize emotion from EEG signals measured with the BraInquiry EEG PET device. He uses a limited number of electrodes and trains a linear classifier based on Fishers discriminant analysis. He considers audio, visual and audiovisual stimuli and trains classifies for positive/negative, aroused/calm and audio/visual/audiovisual.

Takahashi [18] use a headband of three dry electrodes to classify five emotions (joy, anger, sadness, fear, and relaxation) based on multiple bio-potential signals (EEG, pulse, and skin conductance). He trains classifiers using support vector machines and reports the resulting classifying accuracy both using the whole set of bio-potential signals, and solely based on EEG signals.

Lin at al. [10] apply machine-learning techniques to categorize EEG signals according to subject self-reported emotional states during music listening. They propose a framework for systematically seeking emotion-specific EEG features and exploring the accuracy of the classifiers. In particular, they apply support vector machines to classify four emotional states: joy, anger, sadness, and pleasure.

\section{Data Collection}

EEG data in this study were collected from 6 healthy subjects (3 males and 3 females) with average age of 30.16 during listening to emotion-annotated sounds. 
For collecting the data we used the Emotiv EPOC headset, recently released by the Emotiv Company [7. This headset consists of 14 data-collecting electrodes and 2 reference electrodes, located and labeled according to the international 10-20 system 12. Following the international standard, the available locations are: AF3, F7, F3, FC5, T7, P7, O1, O2, P8, T8, FC6, F4, F8 and AF4. Figure 2 shows the 14 Emotiv EPOC headset electrode positions. The EEG signals are transmitted wirelessly to a laptop computer.

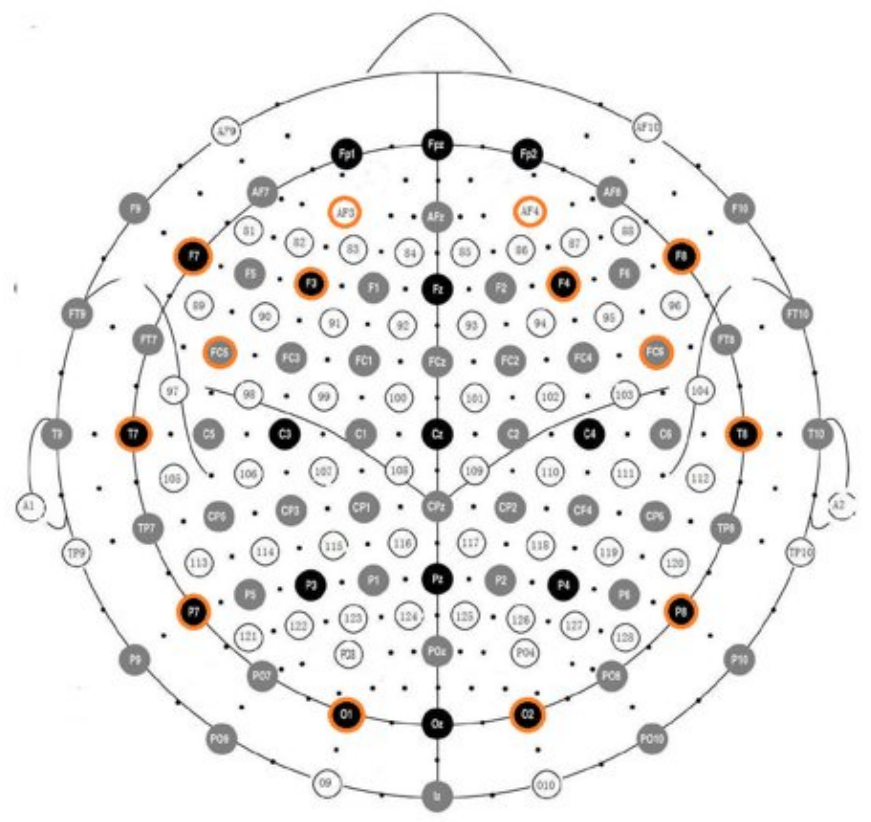

Fig. 2. Emotiv EPOC headset electrode positioning

Subjects were instructed to look at a cross in the a computer screen and to remain seated during the experiment. Subjects listened to selected sounds from the IADS library of emotion-annotated sounds 1 which is available for emotion research. Based on the annotations provided by the stimuli databases, we selected 12 sound stimuli situated in the extremes on the arousal-valence emotion plane: three positive/aroused, three positive/calm, three negative/calm, and three negative/aroused. The stimuli were selected to be as much as possible on the extremes of the two-dimensional emotion plane and as unanimous as possible, since we do not consider self-reporting information to cater for persondependent deviations.

Initially, the subjects are informed about the experiment procedure and instructed to follow the usual guidelines during stimuli presentation (e.g. do not blink or move). Once this was done, 12 sound stimuli are randomly presented each one for five seconds and a 10 second silent rest is inserted between stimuli. 
The purpose of the 10 second silent rests is to set a neutral emotional state of mind in between stimuli.

\section{Data Classification}

\subsection{Feature Extraction}

As mentioned in the previous section, in EEG signals the alpha $(8-12 \mathrm{~Hz})$ and beta $(12-30 \mathrm{~Hz})$ bands are particular bands of interest in emotion research for both valence and arousal 13. The presence of EOG artifacts (eye movement/blinking) is most dominant below $4 \mathrm{~Hz}$, ECG (heart) artifacts around $1.2 \mathrm{~Hz}$, and EMG (muscle) artifacts above $30 \mathrm{~Hz}$. Non physiological artifacts caused by power lines are normally present above $50 \mathrm{~Hz}$ [6 6 ].

Thus, fortunately a byproduct of extracting the alpha and beta frequencies is that much of the noise present in EEG signals is considerably reduced. We apply bandpass filtering for extracting alpha and beta frequency bands. Using Fourier frequency analysis, the original signal is split up in frequencies in order to remove specific frequencies, before transforming back the signal with only the frequencies of interest. For this research, we apply the bandpass filter implementation provided by the OpenVibe software [15].

From the EEG signal of a person, we determine the level of arousal, i.e. how relaxed or excited the person is, by computing the ratio of the beta and alpha brainwaves as recorded by the EEG. We measure the EEG signal in four locations (i.e. electrodes) in the prefrontal cortex: AF3, AF4, F3 and F4 (see Figure 2). As mentioned before, beta waves are associated with an alert or excited state of mind, whereas alpha waves are more dominant in a relaxed state. Alpha activity has also been associated to brain inactivation. Thus, the beta/alpha ratio is a reasonable indicator of the arousal state of a person.

In order to determine the valence level, i.e. negative or positive state of mind, we compare the activation levels of the two cortical hemispheres. This is motivated by psychophysiological research which has shown the importance of the difference in activation between the cortical hemispheres. Left frontal inactivation is an indicator of a withdrawal response, which is often linked to a negative emotion. On the other hand, right frontal inactivation may be associated to an approach response, or positive emotion.

As mentioned before, high alpha activity is an indication of low brain activity, and vice versa. Thus, an increase in alpha activity together with a decrease in beta waves may be associated with cortical inactivation [13. F3 and F4 are the most used positions for looking at this alpha activity, as they are located in the prefrontal lobe which plays a crucial role in emotion regulation and conscious experience.

Although previous research suggests that hemispherical differences are not an indication of affective valence (feeling a positive or negative emotion), it has been suggested that it is an indication of motivational direction (approach or withdrawal behavior to the stimulus) 8 . In general, however, affective valence is related to motivational direction. Therefore, comparing hemispherical activation 
seems to be a reasonable method to detect valence. Thus, we estimate the valence value in a person by computing and comparing the alpha power $a$ and beta power $b$ in channels F3 and F4. Specifically,

$$
\text { valence }=a_{F 4} / b_{F 4}-a_{F 3} / b_{F 3} \text {. }
$$

\subsection{Learning Task}

In this section we describe our approach to training and evaluating classifiers for the task of detecting the emotional state of mind of a person given the person's observed EEG data. We approach this problem as a two 2-class classification problem. In particular, we apply machine learning techniques to classify high/low arousal and positive/negative valence emotional states. The obtained classifiers can be used to classify emotions such as happiness, anger, sadness, and calm. Figure 3 shows these emotions in the arousal/valence plane.

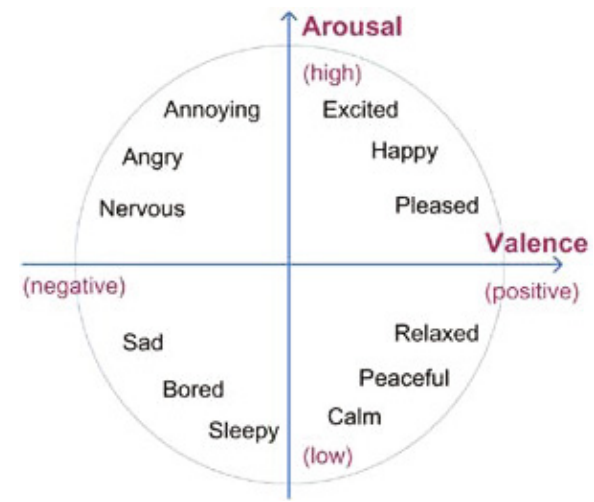

Fig. 3. Emotional states and their positions in the valence/arousal plane

We are interested in inducing two classifiers of the following forms:

$$
\text { ArousalClassifier }(\text { EEGdata }([t, t+c])) \rightarrow\{\text { high, low }\}
$$

and

$$
\text { ValenceClassifier }(\text { EEGdata }([t, t+c])) \rightarrow\{\text { positive, negative }\}
$$

where EEGdata $([t, t+c])$ is the EEG data observed at time interval $[t, t+c]$ and $\{$ high,low and $\{$ positive, negative $\}$ are the sets of emotional states to be discriminated. The results reported in this paper are obtained with $c=1 \mathrm{~s}$ and with increments of $t$ of $0.0625 \mathrm{~s}$. For each subject in the EEG data sets we train a separate classifier. 


\subsection{Algorithms}

In this paper we evaluate two classifiers, Linear Discriminant Analysis (LDA) [1] and Support Vector Machines (SVM) [4, for classifying an emotion state for each EEG segment. Linear discriminant analysis and the related Fisher's linear discriminant are methods used in statistics, pattern recognition and machine learning to find a linear combination of features which characterizes or separates two or more classes of objects or events. The resulting combination may be used as a linear classifier. LDA is closely related to regression analysis, which also attempt to express one dependent variable as a linear combination of other features. In regression analysis however, the dependent variable is a numerical quantity, while for LDA it is a categorical variable (i.e. the class label).

On the other hand, SVM is one of the most popular supervised learning algorithms for solving classification problems. The basic idea in SVM is to project input data onto a higher dimensional feature space via a kernel transfer function, which is easier to be separated than that in the original feature space. Depending on input data, the iterative learning process of SVM would eventually converge into optimal hyperplanes with maximal margins between each class. These hyperplanes would be the decision boundaries for distinguishing different data clusters. Here, we use linear and radial basis function (RBF) kernel to map data onto a higher dimension space. The results reported are obtained using the LDA and SVM implementations in the OpenVibe software [15.

We evaluated each induced classifier by performing the standard 10 -fold cross validation in which $10 \%$ of the training set is held out in turn as test data while the remaining $90 \%$ is used as training data. When performing the 10-fold cross validation, we leave out the same number of examples per class. In the data sets, the number of examples is the same for each class considered, thus by leaving out the same number of examples per class we maintain a balanced training set.

\subsection{Results}

Given that we are dealing with 2-class classification tasks and that the number of instances in each class is the same, the expected classification accuracy of the default classifier (one which chooses the most common class) is $50 \%$ (measured in correctly classified instances percentage). For the high-versus-low arousal, and the positive-versus-negative valence classifiers the average accuracies obtained for SVM with radial basis function kernel classifier were $77.82 \%$, and $80.11 \%$, respectively. For these classifiers the best subject's accuracies were $83,35 \%$, and $86.33 \%$, respectively. The correctly classified instances percentage for each subject and each learning method is presented in Figures 4 and 5 .

\subsection{Discussion}

The difference between the results obtained and the accuracy of a baseline classifier, i.e. a classifier guessing at random confirms that the EEG data contains sufficient information to distinguish between high/low arousal and positive/negative 


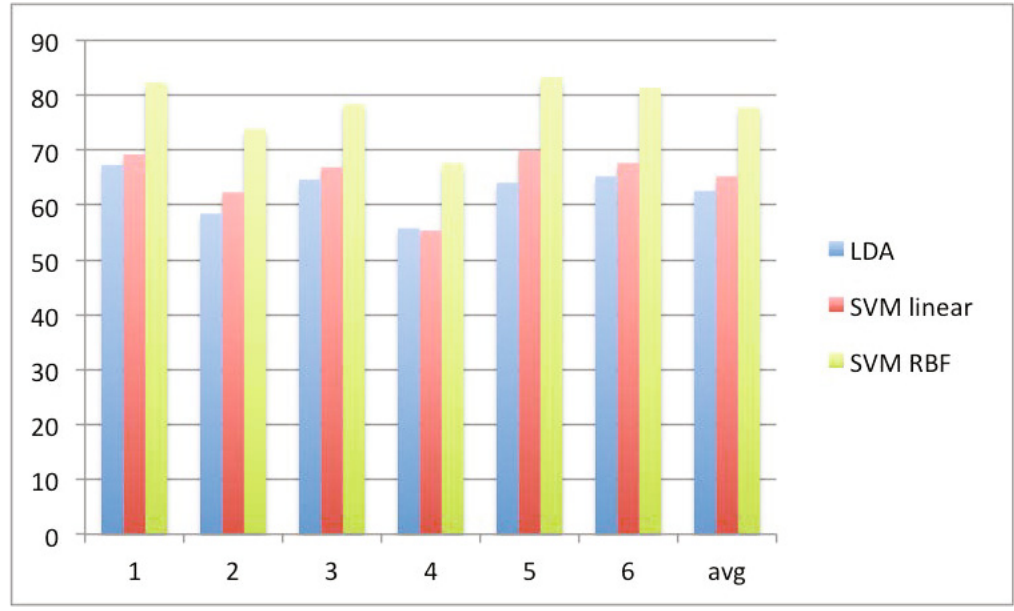

Fig. 4. Classifiers (LDA, SVM with linear kernel, and SVM with radial basis function kernel) accuracies for high-versus-low arousal for all subjects

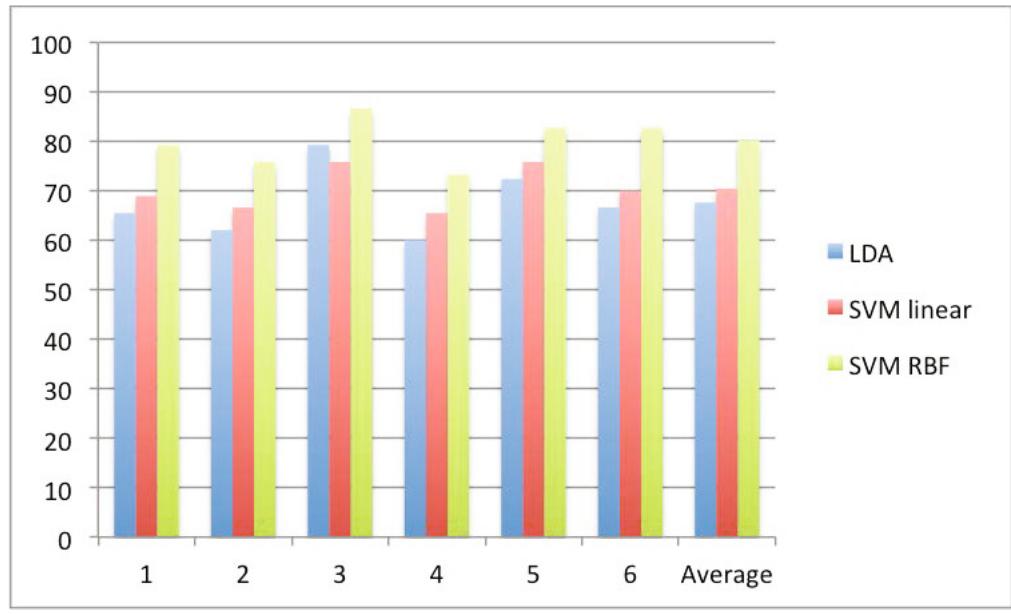

Fig. 5. Classifiers (LDA, SVM with linear kernel, and SVM with radial basis function kernel) accuracies for positive-versus-negative valence for all subjects

valence states, and that machine learning methods are capable of learning the EGG patterns that distinguish these states. It is worth noting that both learning algorithm investigated (LDA and SVM) produced better than random classification accuracies. This supports our statement about the feasibility of training classifiers using the Emotiv Epoc for the tasks reported.

The accuracy of the classifiers for the same task for different subjects varies significantly, even using the same learning method. Subjects producing high 
accuracies with one learning method tend to produce high accuracies with the other learning methods. These uneven accuracies among subjects may be due to different degrees of emotional response between different individuals, or to the amount of noise for different subjects. In any case, it has been reported that there exists considerable variation in EEG responses among different subjects.

It is worth mentioning that in all the experiments performed we provided no self-assessment information about the emotional states by the subjects. This contrasts with other approaches (e.g. [10]) where EEG data is categorized according to subject self-reported emotional states. Incorporating self-assessment information would very likely improve the accuracies of the classifiers.

\section{Conclusion}

We have explored and compared two machine learning techniques for the problem of classifying the emotional state of a person based on EEG data using the Emotiv Epoc headset. We considered two machine learning techniques: linear discriminant analysis and support vector machines. We presented the results of the induced classifiers which are able to discriminate between high-versuslow arousal and positive-versus-negative valence. Our results indicate that EEG data obtained with the Emotiv Epoc device contains sufficient information to distinguish these emotional states, and that machine learning techniques are capable of learning the patterns that distinguish these states. Furthermore, we proved that it is possible to train successful classifiers with no to self-assessment of information about the emotional states by the subjects. As future work, we are particularly interested in systematically exploring different feature extraction methods and learning methods in order to improve the accuracy of the induced classifiers.

Acknowledgments. This work is supported by the Spanish TIN project DRIMS (TIN2009-14274-C02-01).

\section{References}

1. Bradley, M.M., Lang, P.J.: International Affective Digitized Sounds (IADS): Stimuli, Instruction Manual and Affective Ratings. The Center for Research in Psychophysiology, University of Florida, Gainesville, FL, USA (1999)

2. Chanel, G., Kronegg, J., Grandjean, D., Pun, T.: Emotion Assessment: Arousal Evaluation Using EEG's and Peripheral Physiological Signals. In: Gunsel, B., Jain, A.K., Tekalp, A.M., Sankur, B. (eds.) MRCS 2006. LNCS, vol. 4105, pp. 530-537. Springer, Heidelberg (2006)

3. Choppin, A.: Eeg-based human interface for disabled individuals: Emotion expression with neural networks. Masters thesis, Tokyo Institute of Technology, Yokohama, Japan (2000)

4. Cristianini, N., Shawe-Taylor, J.: An Introduction to Support Vector Machines. Cambridge University Press (2000) 
5. Coburn, K., Moreno, M.: Facts and artifacts in brain electrical activity mapping. Brain Topography 1(1), 37-45 (1988)

6. Fatourechi, M., Bashashati, A., Ward, R.K., Birch, G.E.: EMG and EOG artifacts in brain computer interface systems: A survey. Clininical Neurophysiology (118), 480-494 (2007)

7. Emotiv Systems Inc. Researchers, http://www.emotiv.com/researchers/

8. Harmon-Jones, E.: Clarifying the emotive functions of asymmetrical frontal cortical activity. Psychophysiology 40(6), 838-848 (2003)

9. Kandel, E.R., Schwartz, J.H., Jessell, T.M.: Principles of Neural Science. Mc Graw Hill (2000)

10. Lin, Y.-P., Wang, C.-H., Jung, T.-P., Wu, T.-L., Jeng, S.-K., Duann, J.-R., Chen, J.-H.: EEG-Based Emotion Recognition in Music Listening. IEEE Transactions on Biomedical Engineering 57(7) (2010)

11. Mika, S., et al.: Fisher Discriminant Analysis with Kernels. In: IEEE Conference on Neural Networks for Signal Processing IX, pp. 41-48 (1999)

12. Niedermeyer, E., da Silva, F.L.: Electroencephalography, Basic Principles, Clinical Applications, and Related Fields, p. 140. Lippincott Williams \& Wilkins (2004)

13. Niemic, C.P.: Studies of emotion: A theoretical and empirical review of psychophysiological studies of emotion. Journal of Undergraduate Research 1, 15-18 (2002)

14. Bos, D.O.: EEG-based Emotion Recognition: The Influence of Visual and Auditory Stimuli

15. OpenViBE: An Open-Source Software Platform to Design, Test, and Use BrainComputer Interfaces in Real and Virtual Environments. MIT Press Journal Presence' 19(1), 35-53 (2010)

16. Partala, T., Jokiniemi, M., Surakka, V.: Pupillary responses to emotionally provocative stimuli. In: ETRA 2000: Proceedings of the 2000 Symposium on Eye Tracking Research \& Applications, pp. 123-129. ACM Press, New York (2000)

17. Picard, R.W., Klein, J.: Toward computers that recognize and respond to user emotion: Theoretical and practical implications. Interacting with Computers 14(2), 141-169 (2002)

18. Takahashi, K.: Remarks on emotion recognition from bio-potential signals. In: 2nd International Conference on Autonomous Robots and Agents, pp. 186-191 (2004) 